\title{
Stability and Hopf Bifurcation Analysis of a Viral Dynamic Model with Time Delay
}

\author{
Ziping $\mathrm{Han}^{1}$, Hui Lv ${ }^{1}$, Changjun Zhou ${ }^{1}$ and Qiang Zhang ${ }^{1,2, *}$ \\ ${ }^{1}$ Key Laboratory of Advanced Design and Intelligent Computing (Dalian University), Ministry of Education, Dalian, China. \\ ${ }^{2}$ School of Computer Science and Technology, Dalian University of Technology, Dalian, China. \\ ${ }^{*}$ Corresponding author
}

\begin{abstract}
In this paper, a new virus nonlinear dynamic model with time delay is proposed. Moreover, its stability, Hopf bifurcation and other dynamical behavior like chaos are studied. It is indicated that if the reproductive ratio is less than one, the infection-free equilibrium is partially asymptotically stable. Analytical and pictorial results show that if the reproductive ratio is greater than one, the combined effect of the reproductive ratio and the time delay is to create a rich dynamical behavior. Observing and analyzing the process from periodic oscillations to chaos can explain the different pathological feature of patients under different treatment stages. Finally, a simulation example is given to illustrate the correctness and assistance of the study on the virus dynamics research.
\end{abstract}

Keywords—virus model; time delay; stability; Hopf bifurcation; uniform bounded

\section{INTRODUCTION}

Over the past ten years, viral infectious disease dynamics [1] research has always been expected to be the key of overcoming all kinds of human infectious diseases. Considering that dynamic models [2] showed dynamic phenomena of rich variety, it's meaningful to establish an accurate mathematical model to control infectious diseases [3] and ultimately eliminating them.

Establishing a system model is no doubt the first step, and various function models have been used to simulate the condition in patient's body [4]. In order to be closer to realistic situation, time-delay systems [5] has become a research boom in recent years. Therefore, bringing the conception of time delay into the virus dynamics model boasts a lot of significance [6].

In this paper, the main contributions are as follows: 1) To satisfy the assumption that both the delay of the immune response and natural metabolism should be considered simultaneously, the new derived viral dynamic model with time delay is therefore more realistic. 2) We use nonlinear system stability theory and Center Manifold theory to precisely come up with the analysis basis and judgment result. 3) Several kinds of figures are demonstrated to clearly exhibit the stability character and complex dynamic behavior of system. The results extend the analysis on delay virus dynamics considered in the other papers and suggest useful methods to control virus infection.

\section{ESTABLISHMENT OF THE VIRUS MODEL}

There has been much experience in mathematical modeling of traditional epidemic and viral dynamics $[7,8]$ to learn from. Considering the fact that the rate viruses changing to infected cells is much higher than the reproductive ratio of the latter $[9,10]$, namely the amount of free virus is simply proportional to the number of infected cells, a reasonable and bold hypothesis that the number of infected cells $y(t)$ can also be considered as a measure of virus load $v(t)$. Thus getting model as follows

$$
\left\{\begin{array}{l}
\dot{x}(t)=\lambda-d x(t)-\beta x(t) y(t) \\
\dot{y}(t)=\beta x(t) y(t)-a y(t)-p y(t) z(t) \\
\dot{v}(t)=k y(t)-u v(t)
\end{array}\right.
$$

Considering that the immune cells work because of the stimulations of infected cells and viruses to the immune response, there will be a certain response time, so it will be more realistic to bring the time delay into this model. By the way, the natural immune cells metabolism will be delayed definitely at the same time. So the new viral time-delay dynamic model shows as follows

$$
\left\{\begin{array}{l}
\dot{x}(t)=\lambda-d x(t)-\beta x(t) y(t) \\
\dot{y}(t)=\beta x(t) y(t)-a y(t)-p y(t) z(t) \\
\dot{z}(t)=c y(t-\tau)-b z(t-\tau)
\end{array}\right.
$$

where the model consists of the number of uninfected cells $x(t)$, the number of infected cells $y(t)$ and the number of immune cells $z(t)$. By the way, the uninfected cells are generated at a rate $\lambda$, die at a rate $d x(t)$ and become infected by the virus at a rate $\beta x(t) y(t)$. The infected cells are generated at a rate $\beta$, die at a rate $a y(t)$ and are killed by the immune cells at a rate $p y(t) z(t)$. The immune cells are generated at a rate $c y(t-\tau)$, die at a rate $b z(t-\tau)$. 


\section{STABILITY ANALYSIS AND EXISTENCE OF HOPF BIFURCATION}

In this Section, the Lemma 1 shown in the reference [11] will be used, and another Lemma will be introduced at first.

Lemma 2 [12] ( Transverse field conditions of Hopf bifurcation) Make $s(\tau)=\xi(\tau)+i \omega(\tau)$ the characteristic root of

$$
\operatorname{det}(J-s I)=s^{3}+A_{1} s^{2}+A_{2} s+\left(B_{1} s^{2}+B_{2} s+B_{3}\right) e^{-s \tau}=0
$$

the Hopf bifurcation exists if it meets $\xi\left(\tau_{0}\right)=0$ and $\omega\left(\tau_{0}\right)=\omega_{0}$ and its first differential of time delay $\tau$ based on characteristic root , namely

$$
\left.\operatorname{Re}\left(\frac{d s}{d \tau}\right)^{-1}\right|_{\tau=\tau_{k}} \neq 0
$$

In the following part. The existence of nonnegative equilibrium and local stability are going to be researched, and by the way, it is easy to verify that the solution of the system is uniformly and ultimately bounded. Two equilibriums can be derived through calculation.

$$
\begin{gathered}
E_{0}=\left(\begin{array}{ccc}
\frac{\lambda}{d} & 0 & 0
\end{array}\right), E_{1}=\left(\begin{array}{lll}
x_{1} & y_{1} & z_{1}
\end{array}\right) \\
x_{1}=\frac{c \lambda}{a d+b \beta z_{1}}, y_{1}=\frac{b z_{1}}{c}, z_{1}=\frac{-(p c d+a b \beta)}{2 b p \beta}+\frac{\sqrt{(p c d+a b \beta)^{2}-4 b c p \beta(a d-\lambda \beta)}}{2 b p \beta}
\end{gathered}
$$

As for the target system, the virus reproductive ratio is $R=\frac{\lambda \beta}{a d}$. If $R<1, E_{0}$, known as uninfected equilibrium point, will be the only positive one. If $R>1$, here comes a new equilibrium point, $E_{1}$, known as the balanced infection equilibrium point, corresponding to a situation where virus and immune cells exist at the same time.

Theorem 3 If $R \leq 1$, the virus uninfected equilibrium point $E_{0}$ is partially asymptotically stable.

Proof. The Jacobian matrix of system at the equilibrium point $E_{0}$ can get us the eigenvalues equation.

$$
D_{(\lambda)}=(d+\lambda)\left(b e^{-\omega \tau}+\lambda\right)\left(-\frac{\lambda \beta}{d}+a+\lambda\right)
$$

a) When reproductive ratio $R=\frac{\lambda \beta}{a d} \leq 1$, eigenvalues are given by

$$
\lambda_{1}=-d<0, \lambda_{2}=-b e^{-\omega \tau}<0, \lambda_{3}=\frac{\lambda \beta}{d}-a<0
$$

It can be concluded that $E_{0}=\left(\begin{array}{lll}\frac{\lambda}{d} & 0 & 0\end{array}\right)$ is partially asymptotically stable.

b) When reproductive ratio $R=\frac{\lambda \beta}{a d}>1$, since there is at least one solution of characteristic equation greater than zero, the equilibrium $E_{0}$ is therefore a saddle point. It is concluded that snap through buckling happened here, namely when $E_{0}$ loses its stability, system jumps to another equilibrium point state. This completes the proof.

In the next part, let's define the coordinate transformation

$$
x_{*}(t)=x(t)-x_{1}, y_{*}(t)=y(t)-y_{1}, z_{*}(t)=z(t)-z_{1}
$$

Note that equilibrium $E_{1}$ has been translated to the origin. The linearization of system translated to the origin is

$$
\left\{\begin{array}{l}
\dot{x}_{*}(t)=-\left(d+\beta y_{1}\right) x_{*}(t)-\beta x_{1} y_{*}(t) \\
\dot{y}_{*}(t)=\beta y_{1} x_{*}(t)-p y_{1} z_{*}(t) \\
\dot{z}_{*}(t)=c y_{*}(t-\tau)-b z_{*}(t-\tau)
\end{array}\right.
$$

The characteristic equation can be described in the following form,

$$
\operatorname{det}(J-s I)=s^{3}+A_{1} s^{2}+A_{2} s+\left(B_{1} s^{2}+B_{2} s+B_{3}\right) e^{-s r}=0
$$

also denoted as equation (3). Since the stability of system depends on how the roots of characteristic equation distribute, we will study the equation by means of Lemma 1 . It is clear to find that conditions (i)-(v) of Lemma 1 are satisfied.

Through characteristic equation,

$$
\tau_{k}^{(j)}=\frac{1}{\omega_{k}}\left[\arcsin \frac{A_{1} B_{2} \omega_{k}^{3}-\left(B_{3}-B_{1} \omega_{k}^{2}\right)\left(\omega_{k}^{3}-A_{2} \omega_{k}\right)}{B_{2}^{2} \omega_{k}^{2}+\left(B_{3}-B_{1} \omega_{k}^{2}\right)^{2}}+2 j \pi\right]
$$

where $k=1,2,3 ; j=0,1, \ldots$, the $\pm \omega_{k}$ are a pair of pure imaginary root of Eq.(6) when $\tau=\tau_{k}^{(j)}$. The critical time delay parameter $\tau_{k}$ and the critical angle frequency $\omega_{k}$ satisfying (7) when $j=0$ are

$$
\tau_{k}=\frac{1}{\omega_{k}}\left[\arcsin \frac{A_{1} B_{2} \omega_{k}^{3}-\left(B_{3}-B_{1} \omega_{k}^{2}\right)\left(\omega_{k}^{3}-A_{2} \omega_{k}\right)}{B_{2}^{2} \omega_{k}^{2}+\left(B_{3}-B_{1} \omega_{k}^{2}\right)^{2}}\right], \omega_{k}=\frac{-C_{1}-\left(\sqrt[3]{Y_{1}}+\sqrt[3]{Y_{2}}\right)}{3}
$$

where

$$
Y_{1,2}=\left(C_{1}^{2}-3 C_{2}\right) C_{1}+3\left(\frac{-\left(C_{1} C_{2}-9 C_{3}\right)}{2} \pm \frac{\sqrt{\left(C_{1} C_{2}-9 C_{3}\right)^{2}-4\left(C_{1}^{2}-3 C_{2}\right)\left(C_{2}^{2}-3 C_{1} C_{3}\right)}}{2}\right)
$$


At the same time, as for the equation in Lemma 1, we can verify the transversality condition below based on Lemma $2[11]$

$$
\left.\operatorname{Re}\left(\frac{d s}{d \tau}\right)^{-1}\right|_{\tau=\tau_{k}} \neq 0
$$

Synthesizing all the Lemmas and Theorems above and Hopf bifurcation theory, we can obtain that,

Theorem 4 As for the system (2) in the case when reproductive ratio $R>1$.

(i) If $C_{3} \geq 0$ and $\Delta \leq 0$, then Eq. $F(\omega)=0$ doesn't have any positive root, the infection equilibrium point $E_{1}$ is asymptotically stable when $\tau \geq 0$.

(ii) If $C_{3}<0$, then Eq. $F(\omega)=0$ has at least one positive root, and we can calculate critical time delay $\tau_{k}$.

a) When $\tau \in\left[0, \tau_{k}\right)$, stability switches occur limited times.

b) When $\tau=\tau_{k}$, a Hopf bifurcation happens to equilibrium point $E_{1}$ of system, and periodic solution appears.

c) Stability switches occur as time delay increases, making system unstable if $\tau>\tau_{k}$.

\section{SimUlation}

In order to study the stability characteristic as shown in Section 3, we perform simulation and verification based on different reproductive ratio and time delay parameters via Matlab. The standard configuration parameters are chosen as [7]. nonlinear dynamic model
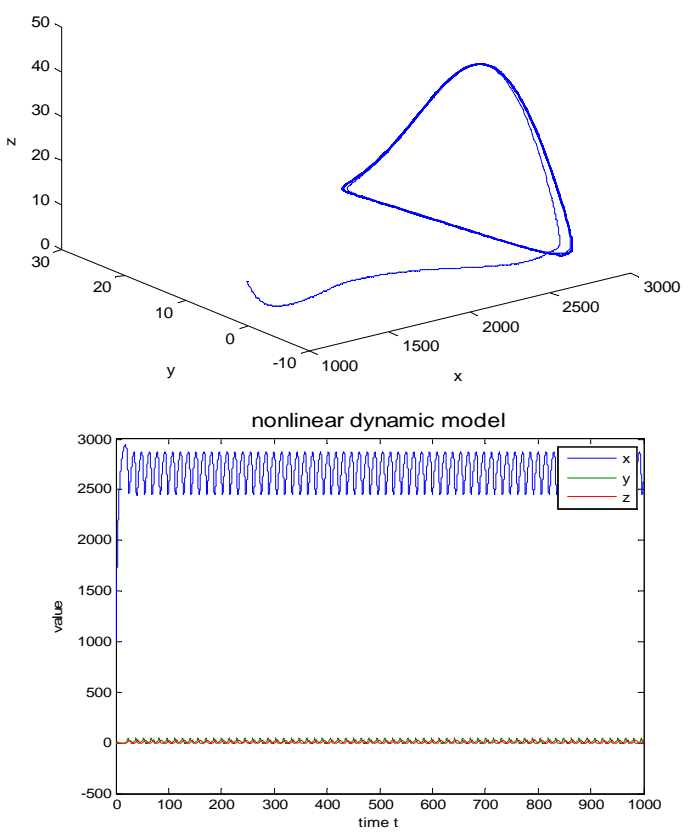

FIGURE I. TIME DELAY $\tau=\tau_{k}=2.22$, REPRODUCTIVE RATIO

$$
R=1.2
$$

nonlinear dynamic model
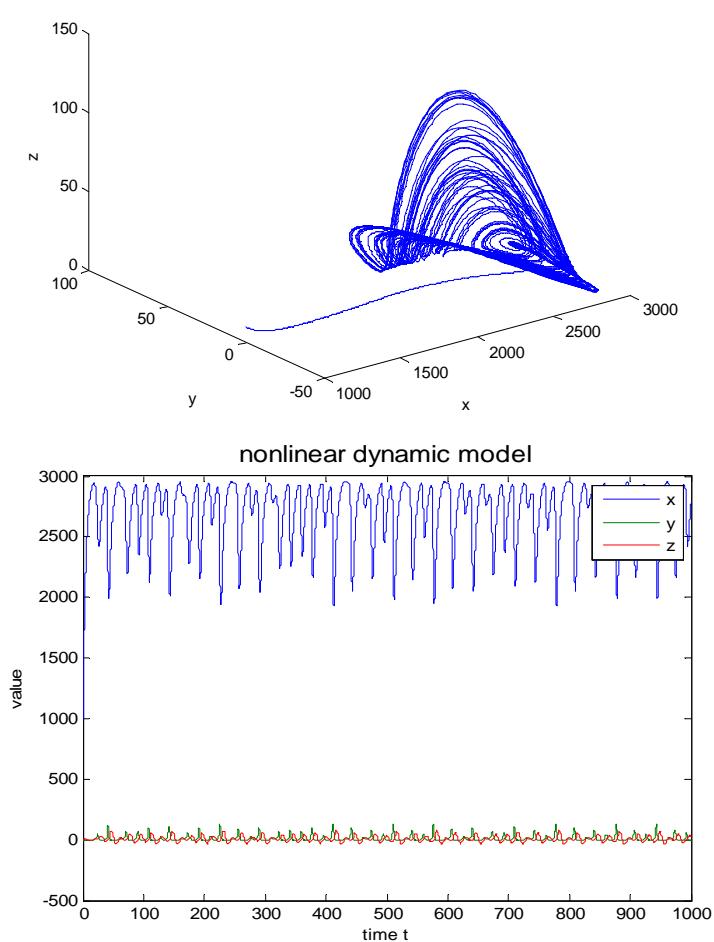

FIGURE II. TIME DELAY $\tau=3.5$, REPRODUCTIVE RATIO $R=1.2$ 
It can be calculated that $\tau_{k}=2.22, \omega_{k}=0.0097$. In the case when $\tau=\tau_{k}=2.22$, Hopf bifurcation happens to virus infected equilibrium point $E_{1}$, as shown in Figure 1.

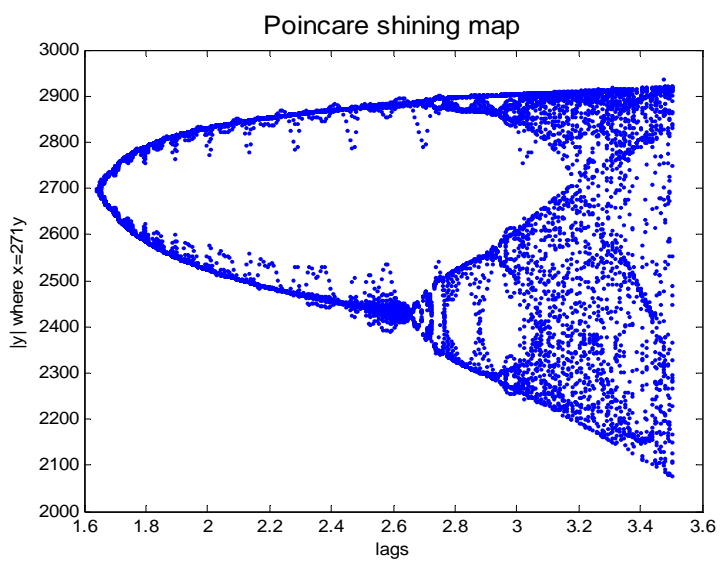

(a)

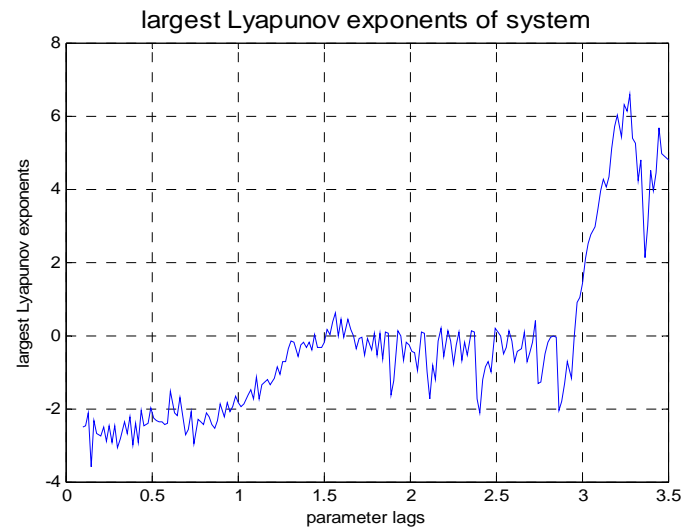

(b)

FIGURE III. REPRODUCTIVE RATIO $R=1.2$. POINCARÈ SHINING MAP FIGURE 3.A, OF WHICH $\tau \in(1.6,3.5)$. THE LARGEST LYAPUNOV EXPONENT FIGURE 3.B, OF WHICH $\tau \in(0,3.5)$

In the period when $\tau>\tau_{k}=2.22$, the virus infection equilibrium point $E_{1}$ is not stable, getting into chaotic state through period-doubling bifurcation, as shown in Figure 2.

From Poincarè shining map Figure 3.a and the largest Lyapunov exponent Figure 3.b, it is obvious to find that the state of equilibrium point has undergone a series of changes due to the increase of the time delay parameter, and it fits precisely the analysis results of the Section 3 in this article.

\section{CONCLUSIONS}

In this article, a new virus nonlinear dynamic model with time delay which could be more realistic is proposed, and its stability, dynamical behavior like Hopf bifurcation. The research has clearly shown that adjusting the cells' reproductive ratio and time delay will affect viral infection. The analysis results will not only help us effectively grasp the complex dynamic behavior, but also provide the theory basis for treatment and operational plan in reality situation. Simulation example and lots of figures have verified the theoretical results given in this article. It is expected that the approach can be further used for the realistic treatment of the infectious diseases.

\section{ACKNOWLEDGEMENTS}

This work is supported by the National Natural Science Foundation of China (Nos.61425002, 61772100, 61702070, 61672121, 61572093, 61402066, 61402067, 61370005, 31370778), Program for Changjiang Scholars and Innovative Research Team in University (No.IRT_15R07), the Program for Liaoning Innovative Research Team in University (No.LT2015002), the Basic Research Program of the Key Lab in Liaoning Province Educational Department (No.LZ2015004).

\section{REFERENCES}

[1] Gandon, S., Day, T., Metcalf, C.J.E., Grenfell, B.T.: Forecasting Epidemiological and Evolutionary Dynamics of Infectious Diseases. Trends in Ecology \& Evolution 31(10), 776-788 (2016).

[2] Tien, M.H., D’Souza, K.: A generalized bilinear amplitude and frequency approximation for piecewise-linear nonlinear systems with gaps or prestress. Nonlinear Dynamics, 88(4), 2403-2416 (2017).

[3] Nakatani, H.: Global Strategies for the Prevention and Control of Infectious Diseases and Non-Communicable Diseases. Journal of Epidemiology 26(4), 171-178 (2016).

[4] Cai, L., Li, X.: Stability and Hopf bifurcation in a delayed model for HIV infection of cells. Chaos, Solitons \& Fractals 42(1), 1-11 (2009).

[5] 5. Park, M.J., Kwon, O.M., Ju, H.P., Lee, S.M., Cha, E.J.: Stability of time-delay systems via Wirtinger-based double integral inequality. Automatica 55(C), 204-208 (2015).

[6] Chen, L., Zhao, T., Li, W., Zhao, J.: Bifurcation control of bounded noise excited Duffing oscillator by a weakly fractional-order [FORMULA] feedback controller. Nonlinear dynamics 83(1-2), 529-539 (2016).

[7] Wang, K., Wang, W., Pang, H., Liu, X.: Complex dynamic behavior in a viral model with delayed immune response. Physica D Nonlinear Phenomena 226(2), 197-208 (2007).

[8] Yang, H., Wei, J.: Analyzing global stability of a viral model with general incidence rate and cytotoxic T lymphocytes immune response. Nonlinear Dynamics 82(1-2), 713-722 (2015).

[9] Bartholdy, C., Christensen, J.P., Wodarz, D., Thomsen, A.R.: Persistent Virus Infection despite Chronic Cytotoxic T-Lymphocyte Activation in Gamma Interferon-Deficient Mice Infected with Lymphocytic Choriomeningitis Virus. Journal of Virology 74(22), 10304-10311 (2000).

[10] Wodarz, D., Christensen, J.P., Thomsen, A.R.: The importance of lytic and nonlytic immune responses in viral infections. Trends in Immunology 23(4), 194-200 (2002).

[11] Cooke, K.L., Pauline, V.D.D.: On zeroes of some transcendental equations. Funkcialaj Ekvacioj 29(1), 77-90 (1986).

[12] Luo, X.S., Chen, G., Wang, B.H., Fang, J.Q.: Hybrid control of period-doubling bifurcation and chaos in discrete nonlinear dynamical systems. Chaos Solitons \& Fractals 18(4), 775-783 (2003). 